\title{
Scatter patterns in lymph node metastases as a novel prognostic indicator in patients with stage III/N2 colorectal cancer
}

\author{
TAKUYA KOIKE $^{1}$, MASAYA MUKAI ${ }^{1}$, SHINICHIRO HIRAIWA ${ }^{2}$, KYOKO KISHIMA $^{1}$, \\ DAIKI YOKOYAMA $^{1}$, SHUJI UDA ${ }^{1}$, SAYURI HASEGAWA ${ }^{1}$, TAKAYUKI TAJIMA ${ }^{3}$, \\ EIJI NOMURA ${ }^{1}$, TOMOKO SUGIYAMA ${ }^{2}$ and TAKUMA TAJIRI ${ }^{2}$ \\ Departments of ${ }^{1}$ Surgery and ${ }^{2}$ Pathology, Tokai University Hachioji Hospital, Tokyo 192-0032; \\ ${ }^{3}$ Department of Surgery, Tokai University Tokyo Hospital, Tokyo 151-0053, Japan
}

Received March 3, 2021; Accepted July 14, 2021

DOI: $10.3892 / \mathrm{mco} .2021 .2402$

\begin{abstract}
To classify patients with stage III/N2 colorectal cancer into high- and low-risk groups for recurrence, the present study compared clinicopathological features by immunohistochemical staining. The single-center analysis included $53 / 668$ patients $(7.9 \%)$ with stage III/N2 colorectal cancer who underwent radical resection between January 2006 and December 2014. The present study examined cancer cell distribution in metastatic lymph nodes and classified patients into a group with circumferential localization patterns like a cystic mass (CLP) and a group with scatter patterns like fireworks (SPF). Subsequently, 5-year relapse-free survival (5Y-RFS) and 5-year overall survival (5Y-OS) rates were compared and the histological type (differentiation degree) of the primary adenocarcinoma was included. The CLP group included 16 patients $(30.2 \%)$ and the SPF group included 37 patients $(69.8 \%)$. The 5Y-RFS rates in these groups were 75.0 vs. $37.8 \%$, respectively $(\mathrm{P}=0.021)$, and the $5 \mathrm{Y}-\mathrm{OS}$ rates were 81.3 vs. $48.6 \%(\mathrm{P}=0.033)$. Patient clinicopathological characteristics exhibited no significant differences between groups. The adenocarcinoma was well differentiated in 14 patients (Well; 26.4\%) and moderately (Mod; $n=37$ ) or poorly (Por; $n=2$ ) differentiated in 39 patients (Mod+Por; 73.6\%). Patients were further classified into four groups: Well/CLP $(n=6)$, Well/SPF $(n=8)$, Mod+Por/CLP $(n=10)$ and Mod+Por/SPF $(n=29)$. For Well/CLP vs. Well/SPF,
\end{abstract}

Correspondence to: Dr Takuya Koike, Department of Surgery, Tokai University Hachioji Hospital, 1838 Ishikawa-machi, Hachioji, Tokyo 192-0032, Japan

E-mail: takuyakoike118@yahoo.co.jp

Abbreviations: 5Y-RFS, 5-year relapse-free survival; 5Y-OS, 5-year overall survival; CLP, circumferential localization patterns like a cystic mass; H\&E, hematoxylin and eosin; L-OHP, oxaliplatin; SPF, scatter patterns like fireworks; AE1/AE3, cytokeratin AE1/AE3

Key words: colorectal cancer, lymph node metastasis, stage III, immunohistochemical staining, cytokeratin staining, AE1/AE3 antibody the 5Y-RFS rates were 66.7 vs. $25.0 \%$, respectively $(\mathrm{P}=0.293)$, and for Mod+Por/CLP vs. Mod+Por/SPF (80.0 vs. 41.4\%; $\mathrm{P}=0.052)$, the respective values for $5 \mathrm{Y}-\mathrm{OS}$ were $66.7 \mathrm{vs.} 50.0 \%$ $(\mathrm{P}=0.552)$ and 90.0 vs. $48.3 \%(\mathrm{P}=0.059)$. Based on the aforementioned results, the CLP group was considered a low-risk group for recurrence with a relatively good prognosis; however, the SPF group was considered a high-risk group for recurrence with a poor prognosis, suggesting a need for more potent multi-combination chemotherapy in these patients from the early postoperative period.

\section{Introduction}

In Japan, the prevalence of colorectal cancer continues to increase with the westernization of lifestyles, and this cancer has become the number one cause of death in women (age-adjusted mortality by site) and the third leading cause of death in men after lung cancer and gastric cancer (1). Patients with stage I/II colorectal cancer, who do not have lymph node metastases, have a relatively good prognosis, but in patients with stage III, who do have lymph node metastases, the 5-year survival rate is only 60 to $70 \%$ and the recurrence rate is 30 to $40 \%$ (2). Patients with stage III/N1, who have 3 or fewer lymph node metastases, have a better prognosis than patients with stage III/N2, and their 5-year survival rate is about $70 \%$ with postoperative multi-combination chemotherapy (2). However, patients with stage III/N2, who have 4 or more lymph node metastases, have a significantly higher recurrence rate, even after the same postoperative adjuvant chemotherapy for the same duration. This higher recurrence rate may be due to individual responses to anticancer drugs, but these drugs are assumed to fail to control potential metastasis or recurrence in patients with multiple lymph node metastases. Nevertheless, in daily clinical practice physicians see patients with stage III/N2 who often survive for 5 years or longer without metastasis or recurrence (low-risk group for recurrence).

In Japan, 6-month adjuvant chemotherapy with a 5-FU-based regimen such as 5FU/LV, FOLFIRI, FOLFOX, and CapeOX, is recommended for stage III colorectal cancer after radical resection (2). More intensive postoperative adjuvant chemotherapy may be required for patients with a poor prognosis (high-risk group for recurrence) who experience 
metastasis or recurrence early after surgery. However, to date few studies have reported on clinical indicators that can be used to classify patients into a high-risk group for recurrence or to determine their prognosis. Pathologists routinely compare and examine distinctive properties of tumor morphology. A number of reports indicate that in particular the degree of tissue differentiation is the most important prognostic factor, and the World Health Organization tumor classification standardizes morphology in the most tumor-infiltrated region from well differentiated to poorly differentiated/others (3). However, tumors have been reported to consist of heterogeneous cell groups with multiple different mutations, and regions with morphologically different degrees of differentiation are known to coexist within the same lesion (4). Therefore, the morphology of metastatic lesions, such as in the lung, liver, and lymph nodes, may reflect the severity of the disease more closely than the morphology of the resected tissue sample from the primary lesion and may thus contribute more to prognosis.

Accordingly, the purpose of this study was to identify new clinical indicators of prognosis in patients with stage III/N2 colorectal cancer by comparing clinicopathological features of primary lesions and lymph node metastases to classify patients into a group with poor prognosis (high-risk group for recurrence) and one with good prognosis (low-risk group for recurrence).

\section{Patients and methods}

Ethics approval. This study was approved by the Institutional Review Board of Tokai University School of Medicine as a cumulative retrospective study (IRB approval no. 20R-137, Kanagawa, Japan).

Patients. From the 668 patients with colorectal cancer who underwent radical resection at Tokai University Hachioji Hospital, Tokyo, Japan, during the approximately 9 years from January 2006 until December 2014, we were able to include 53 patients $(7.9 \%)$ with 4 or more lymph node metastases (N2) and 5-year survival data (Table I). All patients had received 6 courses of postoperative adjuvant chemotherapy with $5 \mathrm{FU} / \mathrm{LV}$ or FOLFOX/CapeOX and oral adjuvant chemotherapy with Cape or UFT/UZEL for 6 months to 1 year (5). In the event of recurrence, margin-negative (R0) resection until no residual cancer was present was performed as quickly as possible, and after the procedure patients were administered 6 courses of chemotherapy with a potent new combination that included a camptothecin-11 drug, such as FOLFIRI/IRIS (5). The 5-year relapse-free survival (5Y-RFS) and 5-year overall survival (5Y-OS) rates were calculated starting from the date of radical resection as specified in the pathology records and electronic medical records and ending with the date when recurrence was confirmed by ultrasonography, computed tomography, or magnetic resonance imaging; patients who died were censored at their date of death, and surviving patients without recurrence were censored on December 31, 2019. The cause of death was based on the ICD10 (Ver.2013) (6). Two patients died from other causes of death. They were both male, in the SPF group, and were in their $60 \mathrm{~s}$. Their causes of death were malignant lymphoma and cerebral stroke. Patients who were transferred to other hospitals during the study period, e.g., because of relocation, were followed up by contacting these hospitals by telephone.

Immunohistochemical staining and assessment. Lymph node specimens were stained with hematoxylin and eosin (H\&E), and formalin-fixed paraffin-embedded tissue was prepared for cytokeratin immunostaining. For this immunohistochemical staining, we used an autostainer (VENTANA BenchMark XT, Roche Diagnostics, Indianapolis, IN, USA) and AE1/3 antibody (VENTANA I-VIEW Pan-cytokeratin kit, Roche Diagnostics), in accordance with the manufacturer's protocol.

Patterns seen after H\&E and immunohistochemical staining were classified as either circumferential localization patterns like a cystic mass (CLP) or scatter patterns like fireworks (SPF). In CLP, cancer cell nests are relatively uniformly and smoothly localized around the edges of the cortex and paracortex of metastatic lymph nodes; the associated tumor secretions and necrotizing substances cause the lymph nodes to inflate and give them the appearance of a cyst (Fig. 1A and B). In SPF, vesicles diffusely infiltrate the lymph node parenchyma and proliferate like fireworks with unclear boundaries toward the inner medulla; they are sometimes accompanied by fibroblast proliferation (Fig. 2A and B). The morphology of metastatic lymph nodes was pathologically evaluated as showing a CLP or SPF scatter pattern by using the following criteria: If the pattern was mostly smooth but had some disturbances around the edges of the cortex or paracortex and the disturbance was observed in more than one quarter of the perimeter, the lymph node was classified as belonging to the CLP group, and any area of infiltration outside the lymph nodes was excluded from the assessment. If at least 1 of 4 or more metastatic lesions showed an SPF pattern, the lymph node was classified as belonging to the SPF group. All assessments were made by a surgeon (KT) and pathologist (HS), both of whom were completely blinded to the clinical information.

We calculated the 5Y-RFS and 5Y-OS rates for the CLP and SPF groups, and compared various clinicopathological features between the groups. Furthermore, we compared the relationship between the scatter pattern of the tumor cells in the metastatic lymph nodes and the histological type (differentiation degree) of the primary lesion. The degree of tumor differentiation of the primary adenocarcinoma was defined on the basis of the largest cut surface containing the most tumor-infiltrated area and was used to classify the patients into 3 groups: Well group, well-differentiated adenocarcinoma $(n=14,26.4 \%)$; Mod group, moderately differentiated adenocarcinoma $(n=37,69.8 \%)$; and Por, poorly differentiated adenocarcinoma $(n=2,3.8 \%)$ (Table I). Among colorectal cancers, well-differentiated adenocarcinoma has a relatively good prognosis compared with moderately and poorly differentiated lesions. Therefore, because only 2 patients were classified into the Por group, we combined the Mod and Por groups into a Mod+Por group $(n=39,73.6 \%)$. Subsequently, we further classified patients into 4 groups (Well/CLP, Well/SPF, Mod+Por/CLP, and Mod+Por/SPF) and calculated the 5Y-RFS and $5 \mathrm{Y}-\mathrm{OS}$ rates of each group.

Statistical analysis. The 5Y-RFS and 5Y-OS rates were calculated by the Kaplan-Meier method, and intergroup comparisons 
Table I. Clinicopathological features of 53 patients with stage III/N2 colorectal cancer.

\begin{tabular}{|c|c|c|c|c|}
\hline Variable & Total cases $(n=53)$ & CLP group $(n=16)$ & SPF group $(n=37)$ & P-value \\
\hline \multicolumn{5}{|l|}{$\operatorname{Sex}, \mathrm{n}(\%)$} \\
\hline Male & $30(56.6)$ & $8(50.0)$ & $22(59.5)$ & \multirow[t]{2}{*}{0.737} \\
\hline Female & $23(43.4)$ & $8(50.0)$ & $15(40.5)$ & \\
\hline Median age at operation, years (range) & $64(38-90)$ & $67(57-85)$ & $62(38-90)$ & 0.123 \\
\hline \multicolumn{5}{|l|}{ Tumor location, n (\%) } \\
\hline Colon & $42(79.2)$ & $14(87.5)$ & $28(75.7)$ & \multirow[t]{2}{*}{0.471} \\
\hline Rectum & $11(20.8)$ & $2(12.5)$ & $9(24.3)$ & \\
\hline \multicolumn{5}{|l|}{ Macroscopic classification $^{\mathrm{a}}, \mathrm{n}(\%)$} \\
\hline Type 1 & $7(13.2)$ & $4(25.0)$ & $3(8.1)$ & \multirow[t]{3}{*}{0.091} \\
\hline Type 2 & $41(77.4)$ & $12(75.0)$ & $29(78.4)$ & \\
\hline Type 3 & $5(9.4)$ & $0(0.0)$ & $5(13.5)$ & \\
\hline \multicolumn{5}{|l|}{ Tumor size, n (\%) } \\
\hline$\leq 20 \mathrm{~mm}$ & $3(5.7)$ & $2(12.5)$ & $1(2.7)$ & \multirow[t]{4}{*}{0.586} \\
\hline $21-50 \mathrm{~mm}$ & $34(64.2)$ & $10(62.5)$ & $24(64.9)$ & \\
\hline $51-100 \mathrm{~mm}$ & $15(28.3)$ & $4(25.0)$ & $11(29.7)$ & \\
\hline$>100 \mathrm{~mm}$ & $1(1.9)$ & $0(0.0)$ & $1(2.7)$ & \\
\hline \multicolumn{5}{|l|}{ Pathological T category $^{\mathrm{a}}, \mathrm{n}(\%)$} \\
\hline $\mathrm{T} 2$ & $7(13.2)$ & $3(18.8)$ & $4(10.8)$ & \multirow[t]{4}{*}{0.620} \\
\hline $\mathrm{T} 3$ & $37(69.8)$ & $12(75.0)$ & $25(67.6)$ & \\
\hline $\mathrm{T} 4 \mathrm{a}$ & $8(15.1)$ & $1(6.3)$ & $7(18.9)$ & \\
\hline $\mathrm{T} 4 \mathrm{~b}$ & $1(1.9)$ & $0(0.0)$ & $1(2.7)$ & \\
\hline \multicolumn{5}{|l|}{ Histological type, n (\%) } \\
\hline Well & $14(26.4)$ & $6(37.5)$ & $8(21.6)$ & \multirow[t]{3}{*}{0.372} \\
\hline Mod & $37(69.8)$ & $10(62.5)$ & $27(73.0)$ & \\
\hline Por & $2(3.8)$ & $0(0.0)$ & $2(5.4)$ & \\
\hline \multicolumn{5}{|l|}{ Lymphatic invasion, n (\%) } \\
\hline ly0 & $1(1.9)$ & $0(0.0)$ & $1(2.7)$ & \multirow[t]{4}{*}{0.203} \\
\hline ly 1 & $30(56.6)$ & $12(75.0)$ & $18(48.6)$ & \\
\hline ly2 & $16(30.2)$ & $2(12.5)$ & $14(37.8)$ & \\
\hline ly3 & $6(11.3)$ & $2(12.5)$ & $4(10.8)$ & \\
\hline \multicolumn{5}{|l|}{ Venous invasion, $\mathrm{n}(\%)$} \\
\hline $\mathrm{v0}$ & $19(35.8)$ & $8(50.0)$ & $11(29.7)$ & \multirow[t]{3}{*}{0.127} \\
\hline $\mathrm{v} 1$ & $27(50.9)$ & $8(50.0)$ & $19(51.4)$ & \\
\hline v2 & 7 (13.2) & $0(0.0)$ & 7 (18.9) & \\
\hline \multicolumn{5}{|l|}{ Pathological N category ${ }^{\mathrm{a}}, \mathrm{n}(\%)$} \\
\hline N2a (LN: 4-6) & $34(64.2)$ & $10(62.5)$ & $24(64.9)$ & \multirow[t]{2}{*}{$>0.999$} \\
\hline N2b (LN: $\geq 7)$ & $19(35.8)$ & $6(37.5)$ & $13(35.1)$ & \\
\hline
\end{tabular}

aJapanese classification of colorectal carcinoma (Eighth edition). The clinicopathological background of the two groups did not show any significant differences for all features. CLP, circumferential localization patterns like a cystic mass surrounded by the lymph node cortex/paracortex; SPF, scatter patterns like fireworks, with patterns that extend into the intraparenchymal lymph node medulla; Well, well differentiated adenocarcinoma; Mod, moderately differentiated adenocarcinoma; Por, poorly differentiated adenocarcinoma; LN, lymph node metastasis.

were performed with the log-rank test. The hazard ratio and 95\% CI were calculated with the Cox proportional hazard regression model. For the analysis of patient demographic factors in the CLP and SPF groups, the Mann-Whitney U test (a nonparametric test) was used for age, and the chi-squared test or Fisher's exact test was used for sex, lymph node metastasis, primary tumor location, macroscopic classification, depth of invasion, histological type, lymphatic vessel invasion, and venous invasion. Each factor was assessed in accordance with the criteria of the Japanese Classification of Colorectal, Appendiceal, and Anal Carcinoma, 8th edition (7). In all tests, a P-value less than 0.05 was considered to be statistically significant. IBM SPSS Statistics for Windows Version 25.0 (IBM Corp, Armonk, NY, USA) was used for statistical analysis. 

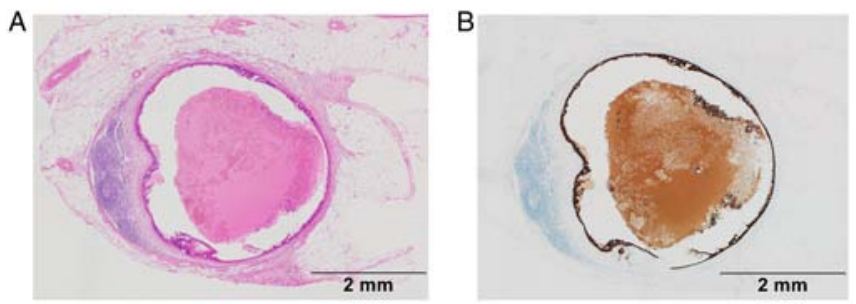

Figure 1. Circumferential localization patterns. (A) H\&E stain (magnification, $\mathrm{x} 40$ ). (B) Cytokeratin AE1/3 stain (magnification, $\mathrm{x} 40$ ). Circumferential localization patterns like a cystic mass surrounded by the lymph node cortex/paracortex.
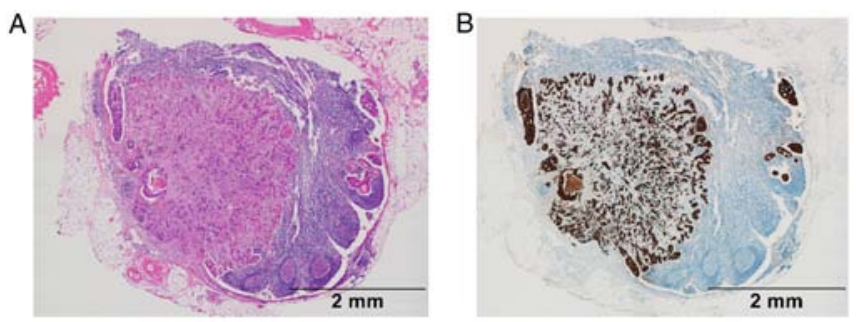

Figure 2. Scatter patterns like fireworks. (A) H\&E stain (magnification, x40) (B) Cytokeratin AE1/3 stain (magnification, $x 40$ ). Scatter patterns like fireworks extending into the intraparenchymal lymph node medulla.

\section{Results}

Follow-up rate. In the follow-up investigation of all patients at 5 years, 30 patients were alive, 22 patients were dead, and 1 patient was lost to follow up, corresponding to a follow-up rate of $98.1 \%$.

Scatter pattern. On the basis of the scatter pattern in the lymph nodes, 16 patients $(30.2 \%)$ were classified into the CLP group and 37 patients $(69.8 \%)$ into the SPF group. In the CLP and SPF groups, the 5Y-RFS rates were 75.0 and $37.8 \%$, respectively $(\mathrm{P}=0.021$; hazard ratio, 3.283; 95\% CI, 1.132-9.519) (Fig. 3A), and the 5Y-OS rates were 81.3 and $48.6 \%$, respectively $(\mathrm{P}=0.033$; hazard ratio, 3.462; 95\% CI, 1.023-11.719) (Fig. 3B). Although the prognosis of the SPF group was significantly worse than that of the CLP group, the comparison of clinicopathological characteristics between these 2 groups showed no significant differences (Table I).

Scatter patterns and tumor differentiation. The 4 groups formed on the basis of the scatter patterns in the metastatic lymph nodes and the degree of tumor differentiation of the primary lesion consisted of the following numbers of patients: Well/CLP, n=6; Well/SPF, n=8; Mod+Por/CLP, n=10; and Mod+Por/SPF, $n=29$. The results of the comparisons of the 5Y-RFS rates between the 2 Well groups and the 2 Mod+Por groups were as follows: $66.7 \%$ (Well/CLP) vs. 25.0\% (Well/SPF) $(\mathrm{P}=0.293$, hazard ratio, 2.361; 95\% CI, 0.475-11.729) and $80.0 \%$ (Mod+Por/CLP) vs. 41.4\% (Mod+Por/SPF) (P=0.052; hazard ratio, 4.295; 95\% CI, 0.990-18.641) (Fig. 4). The 5Y-OS rates between the 2 Well groups and the $2 \mathrm{Mod}+$ Por groups were $66.7 \%$ vs. $50.0 \%(\mathrm{P}=0.552$; hazard ratio, $1.674 ; 95 \% \mathrm{CI}$, $0.306-9.142)$ and $90.0 \%$ vs. $48.3 \%(\mathrm{P}=0.059$; hazard ratio, 7.018; 95\% CI, 0.925-53.217) (Fig. 5).

\section{Discussion}

This study aimed to identify new clinical indicators of prognosis in patients with stage III/N2 colorectal cancer by comparing clinicopathological features of primary lesions and lymph node metastases. We agree that the sample size was limited, and a larger sample size would be preferable. However, this is a single-center analysis, and the number of patients at the study stage was limited, approximately 15 patients annually. Accordingly, it was difficult to include additional patients. Instead, we attempted to improve the follow-up rate. We expect that we will obtain more patients for analysis in the future. The results showed that patients with a CLP scatter pattern in lymph node metastases have a low risk for recurrence and relatively good prognosis, whereas patients with an SPF scatter pattern have a high risk for recurrence and a relatively poor prognosis.

In Japan, patients with stage III colorectal cancer who undergo radical resection, such as total mesocolic excision or total mesorectal excision, have a relatively good prognosis compared with patients in Europe and the United States (1). Patients with stage III colorectal cancer are generally treated with a 6-month postoperative 5-FU-based adjuvant chemotherapy with dexamethasone, imatinib, and vincristine; however, potent chemotherapy such as oxaliplatin (L-OHP) administered by the intravenous route is recommended for patients at high risk for recurrence. The National Comprehensive Cancer Network Clinical Practice Guidelines in Oncology also recommend regimens such as FOLFOX, FLOX, and CapeOX, including L-OHP, for postoperative stage III colon cancer $(8,9)$. However, in a relatively large number of patients these potent chemotherapies increase the frequency and duration of side effects such as peripheral neuropathy, and the guidelines from the Japanese Society for Cancer of the Colon and Rectum (2) do not include supportive evidence recommending these chemotherapies for all stage III patients. Currently, the decision about chemotherapy is often left to the physician and is based on the wishes of the patient and their family; when deciding on treatment options, physicians consider the patient's age, performance status, compliance, the presence or absence of comorbidities, and the benefits and disadvantages for the patient.

In our department, we proactively give FOLFOX/CapeOX as an adjuvant chemotherapy after the surgical procedure in patients with stage III/N2 colorectal cancer who are aged 75 years or younger and have a performance status of 0 to 2 . In accordance with this, 37 (69.8\%) of 44 patients aged 75 years or younger in the target group of this study were given chemotherapy that included L-OHP. However, the results of this study indicated that the prognosis of the SPF group was extremely poor. Accordingly, it may be necessary to conduct a study accumulating data on patients at high risk of recurrence who are given more potent multi-combination chemotherapies used for stage IV colorectal cancer, including molecular targeted drugs such as vascular endothelial growth factor/epidermal growth factor receptor inhibitors, from the early postoperative period. We also consider that investigation of data from multiple institutions and large geographic areas is necessary.

Various studies on factors for poor prognosis in colorectal cancer identified patients with a poor prognosis 
A

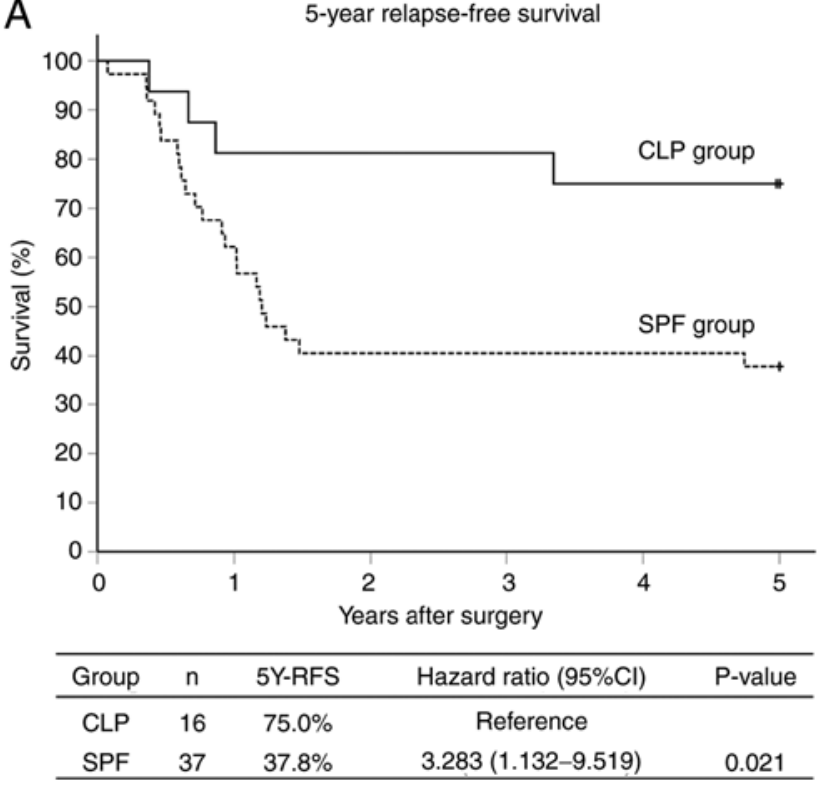

B

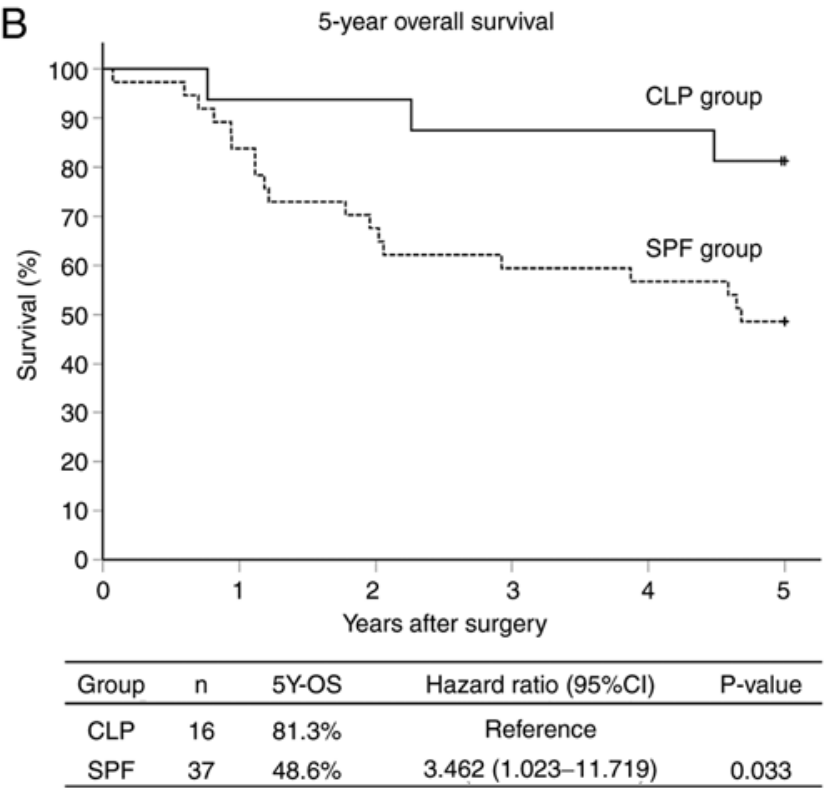

Figure 3. Kaplan-Meier curves. (A) 5Y-RFS by scatter patterns in metastatic lymph nodes. The 5Y-RFS rate was 75.0\% in the CLP group (n=16) and $37.8 \%$ in the $\mathrm{SPF}$ group $(\mathrm{n}=37 ; \mathrm{P}=0.021$; hazard ratio, 3.283; $95 \% \mathrm{CI}, 1.132-9.519)$. (B) $5 \mathrm{Y}-\mathrm{OS}$ by scatter patterns in metastatic lymph nodes. The $5 \mathrm{Y}-\mathrm{OS}$ rate was $81.3 \%$ in the CLP group $(\mathrm{n}=16)$ and $48.6 \%$ in the SPF group ( $\mathrm{n}=37$; $\mathrm{P}=0.033$; hazard ratio, 3.462; 95\% CI, 1.023-11.719). 5Y-OS, 5-year overall survival; 5Y-RFS, 5-year relapse-free survival; 95\% CI, 95\% confidence interval; CLP, circumferential localization patterns like a cystic mass surrounded by the lymph node cortex/paracortex; SPF, scatter patterns like fireworks into the intraparenchymal lymph node medulla.

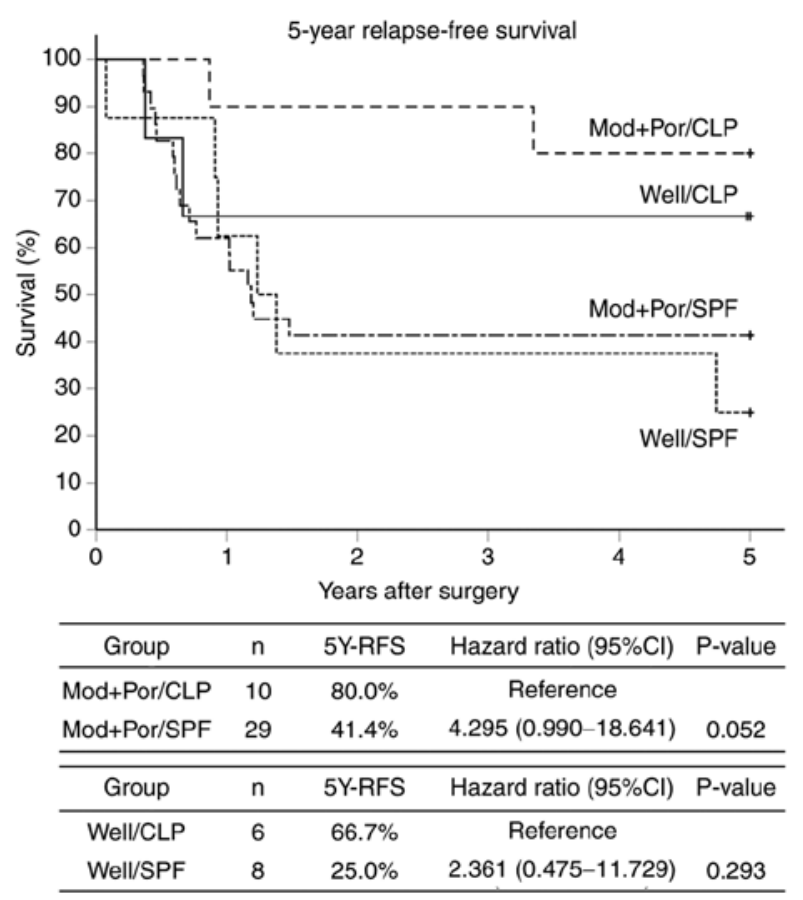

Figure 4. Kaplan-Meier curves 5Y-RFS by histological type of primary tumors and scatter patterns in metastatic lymph nodes. Patients were divided into four subgroups on the basis of the scatter pattern (CLP or SPF) and the differentiation of the primary lesion (Well or Mod+Por) as follows: Well/CLP group ( $n=6)$, Well/SPF group $(n=8)$, Mod+Por/CLP group $(\mathrm{n}=10)$ and Mod+Por/SPF group $(\mathrm{n}=29)$. The 5 Y-RFS rates in the Well/CLP vs. Well/SPF groups were 66.7 vs. $25.0 \%$, respectively $(\mathrm{P}=0.293$; hazard ratio, 2.361; 95\% CI, 0.475-11.729), and those in the Mod+Por/CLP vs. Mod+Por/SPF group were 80.0 vs. $41.4 \%$, respectively $(\mathrm{P}=0.052$; hazard ratio, 4.295; 95\% CI, 0.990-18.641). 5Y-RFS, 5-year relapse-free survival; 95\% CI, 95\% confidence interval; CLP, circumferential localization patterns like a cystic mass surrounded by the lymph node cortex/paracortex; SPF, scatter patterns like fireworks into the intraparenchymal lymph node medulla; Well, well-differentiated adenocarcinoma; Mod+Por, moderately or poorly differentiated adenocarcinoma.

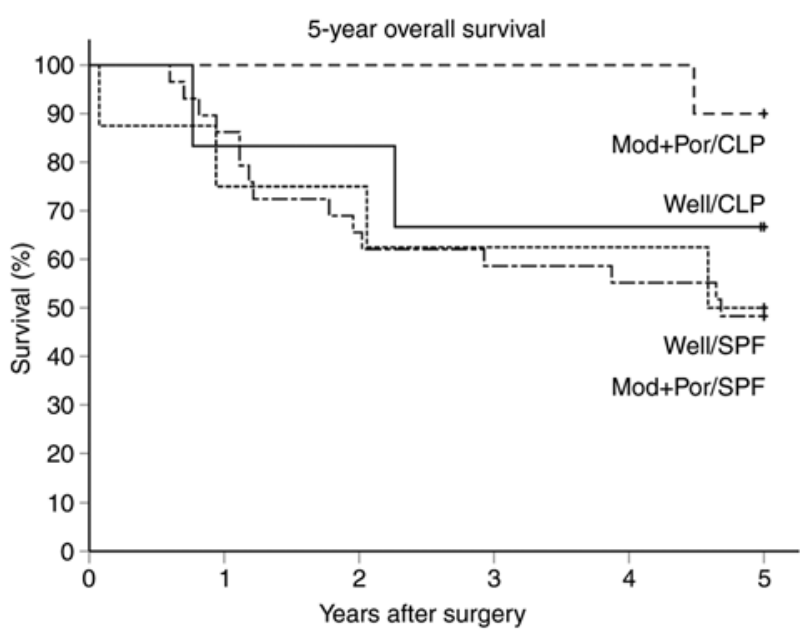

\begin{tabular}{ccccc}
\hline Group & $\mathrm{n}$ & $5 \mathrm{Y}$-OS & Hazard ratio $(95 \% \mathrm{Cl})$ & P-value \\
\hline Mod+Por/CLP & 10 & $90.0 \%$ & Reference & \\
Mod+Por/SPF & 29 & $48.3 \%$ & $7.018(0.925-53.217)$ & 0.059 \\
\hline Group & $\mathrm{n}$ & $5 \mathrm{Y}$-OS & Hazard ratio $(95 \% \mathrm{Cl})$ & P-value \\
\hline Well/CLP & 6 & $66.7 \%$ & Reference & \\
Well/SPF & 8 & $50.0 \%$ & $1.674(0.306-9.142)$ & 0.552 \\
\hline
\end{tabular}

Figure 5. Kaplan-Meier curves 5Y-OS by histological type of primary tumors and scatter patterns in metastatic lymph nodes. Patients were divided into four subgroups on the basis of the scatter pattern (CLP or SPF) and the differentiation of the primary lesion (Well or Mod+Por) as follows: Well/CLP group $(n=6)$, Well/SPF group $(n=8)$, Mod+Por/CLP group $(n=10)$ and Mod+Por/SPF group $(\mathrm{n}=29)$. The $5 \mathrm{Y}-\mathrm{OS}$ rates in the Well/CLP vs. Well/SPF groups were 66.7 vs. $50.0 \%$, respectively ( $\mathrm{P}=0.552$; hazard ratio, $1.674 ; 95 \% \mathrm{CI}, 0.306-9.142$ ); and those in the Mod+Por/CLP vs. Mod+Por/SPF group were 90.0 vs. $48.3 \%$, respectively $(\mathrm{P}=0.059$; hazard ratio, 7.018; 95\% CI, 0.925-53.217). 5Y-OS, 5-year overall survival; 95\% CI, 95\% confidence interval; CLP, circumferential localization patterns like a cystic mass surrounded by the lymph node cortex/paracortex; SPF, scatter patterns like fireworks into the intraparenchymal lymph node medulla; Well, well-differentiated adenocarcinoma; Mod+Por, moderately or poorly differentiated adenocarcinoma. 
by various methods, including pathomorphological lymph node evaluation $(10,11)$, the number of lymph node dissections $(12,13)$, the ratio of the number of metastatic lymph nodes to the number of dissected lymph nodes (13-16), and tumor deposits (17-19). Some also evaluated malignancy by performing immunohistochemical staining to detect micrometastases of lymph nodes that cannot be shown by H\&E staining (20-23). At our hospital, we have been using cytokeratin immunostaining (AE1/3) to detect free cancer cells floating in the lymph sinusoids in dissected lymph nodes from patients with various types of cancer, such as colorectal cancer, gastric cancer, and lung cancer, and have often reported on the relationship with prognosis (24-30). We have used immunostaining to detect free cancer cells, which are difficult to evaluate by $H \& E$ staining, and have found that these cells are useful for identifying a group with poor prognosis among patients with a high recurrence rate (24-31). However, recent studies reported that these free cancer cells are becoming difficult to detect even in patients with more advanced stage III cancers, and researchers assume that these cells are being efficiently eliminated by potent chemotherapies being used recently. Because some patients have a poor prognosis whatever the stage of their cancer, we need to search for new factors that can predict resistance to chemotherapy.

In this study, we examined whether prognosis can be determined on the basis of the assumption that metastatic lesions may be more useful for prognosis than the primary lesion because of the heterogeneous properties of the latter. Metastatic lesions can indicate the potential to metastasize, infiltrate, and proliferate in other tissues, as well as the potential for cancer cells to remain in the body after the primary lesion is surgically removed. By applying our immunostaining method on lymph node tissue, we performed a preliminary analysis in more than 100 patients with stage III/N1, III/N2, and stage IV colorectal cancer and found that metastatic lymph nodes can be morphologically classified into 2 main groups: CLP and SPF. Also, we were able to unify and stabilize evaluations among diagnosticians by using cytokeratin immunostaining (AE1/3) to clearly visualize the morphology of the metastases, including micrometastases, in tissue from metastatic lymph nodes identified by H\&E staining.

In stage III colorectal cancer, studies have found a large difference in the prognosis of patients with stage N1 and those with stage N2. Furthermore, the survival curve of some patients with stage $\mathrm{N} 2$ was reported to be similar to that observed in patients with stage IV cancer (14). In our preliminary analysis, 93 patients $(13.9 \%)$ had stage III/N1 cancer; 43 of these patients $(46.2 \%)$ were classified into the CLP group and 50 patients $(53.8 \%)$ into the SPF group. The 5Y-RFS rates were 66.0 and $62.8 \%(\mathrm{P}=0.709)$, respectively, and the 5Y-OS rates were 76.0 and $74.4 \%(\mathrm{P}=0.761)$, respectively (data not shown). The lack of a statistically significant difference between the CLP and SPF groups was thought to be due to the findings that many patients with stage III/N1 cancer have a good prognosis, the survival rate of the population is higher than that of the population with stage N2 cancer, and recurrence is rarely observed in patients with stage N1 cancer. Also, the worse prognosis of patients with stage N2 was considered to be due to the higher tumor occupation and tumor volume in lymph node metastases and the greater number of lymph node metastases compared with patients with stage N1.

In general, more than 70 to $80 \%$ of the primary lesions of colorectal cancer are histologically classified as well or moderately differentiated adenocarcinoma; therefore, hematogenous, distant metastases or recurrence in the lung or liver is fatal in many of these patients, and predicting prognosis by the histological type of the primary lesion is considered to be difficult (32). When we compared the differentiation of the primary lesions and the metastatic lymph node tumor cells in our study, most of the primary lesions (51/53 patients, 96.2\%) were classified as differentiated adenocarcinoma (Well, $n=14$; Mod, $n=37$ ). In the SPF group, evidence of poor differentiation, which was equivalent to poorly differentiated adenocarcinoma, was observed in $57.1 \%$ (8/14 patients), and in many cases the histological types of the primary lesion and metastatic lymph node tumor did not match. Therefore, we assumed that the metastatic properties of the primary tumor tissue caused the metastatic lesion to differ from the primary tumor, depending on its site and localization, and decreased the amount of differentiation in the metastases. In our study, 8 patients in the Well/SPF group had a poor prognosis with a 5 Y-RFS rate of $25.0 \%$ and a 5 Y-OS rate of $50 \%$, even though well-differentiated lesions usually have a relatively good prognosis. Therefore, these findings suggest that evaluating metastatic lymph nodes can reveal a poor prognosis that would not be assumed by analyzing histological type alone.

Compared with the CLP pattern, the SPF pattern was assumed to indicate that the tumor was more destructive to tissues and had higher infiltration and proliferation capacities. The malignancy in the patients with multiple lymph node metastases (Total number $\geq 4$ ) was assumed to be closely related to the lower degree of tissue differentiation $(33,34)$. We assumed that the groups of tumor cells present in these metastatic lesions remained in the body after surgical resection of the primary lesion and that they were likely to induce metastasis and recurrence. Therefore, molecular targeted therapy may be effective if given after searching for genes such as RAS and BRAF in tumor cells from these metastatic lesions. In the CLP group, we assumed that the tumors had metastasis and engraftment capacities but lacked the infiltration and proliferation capacities that increase tumor volume in the lymph nodes and cause poor differentiation. As mentioned above, the degree of scattering at metastatic loci predicts poor prognosis and is considered to be affected by tumor immunity (35). Thus, in patients with the CLP pattern, limited portions of the gland ducts become filled with secretions and necrotic substances, expand, and become cystic. We expect that in the future this phenomenon of cystic lymph nodes will be clarified by genetic and molecular biological studies as a clinical indicator of low risk for recurrence.

Currently, we are comparing CLP and SPF groups in patients with other gastrointestinal cancers, such as esophageal cancer and gastric cancer, which are also likely to metastasize to the lymph nodes, and we are trying to eliminate the difference in diagnosis among examiners by simply classifying 
the metastases by H\&E staining to quantify them more easily. Because the lymph nodes contain an extremely large number of lymphocytes with a normal genome, the amount of tumor-derived genome is likely to be high. Therefore, in the future a technology is needed that can extract the genomic information of tumor cells by examining potentially fatal distant metastases in the lung, liver, and other organs.

In conclusion, we performed a pathomorphological evaluation of the relationship between metastatic lymph nodes of patients with stage III/N2 colorectal cancer and prognosis by using cytokeratin immunostaining. The CLP group was found to be at low risk for recurrence and to have a relatively good prognosis; however, the SPF group was found to be at high risk for recurrence and to have a poor prognosis, indicating that patients with this pattern on immunostaining require more potent multi-combination chemotherapy from the early postoperative period.

\section{Acknowledgements}

Not applicable.

\section{Funding}

No funding was received.

\section{Availability of data and materials}

The datasets used and/or analyzed during the current study are available from the corresponding author on reasonable request.

\section{Authors' contributions}

TK, MM and SHi performed the research for the present study, contributed to the data analysis, and wrote the manuscript. SHi and MM designed the protocol for the present study, provided surgical advice and supervised the present study. KK, DY, SU, SHa, TakaT, TS and TakuT analyzed the data. TK and SHi diagnosed and assessed the pathological findings. EN acquired the surgical data. TK, KK and $\mathrm{SHi}$ confirm the authenticity of all the raw data. TakuT supervised the study and critically reviewed the manuscript. All authors read and approved the final manuscript.

\section{Ethics approval and consent to participate}

Ethics approval was provided by the Research and Study Program of Tokai University Educational System General Research Organization (IRB approval no. 20R-137). Written informed consent to participate was obtained from all patients.

\section{Patient consent for publication}

Written informed consent for publication of the present study was obtained from the patients.

\section{Competing interests}

The authors declare that they have no competing interests.

\section{References}

1. Ministry of Health, Labor and Welfare: Vital Statistics Japan. Ministry of Health, Labor and Welfare, Tokyo, 2019. https:// ganjoho.jp/reg_stat/statistics/dl/index.html\#mortality (In Japanese).

2. Hashiguchi Y, Muro K, Saito Y, Ito Y, Ajioka Y, Hamaguchi T, Hasegawa $\mathrm{K}$, Hotta $\mathrm{K}$, Ishida $\mathrm{H}$, Ishiguro M, et al: Japanese society for cancer of the colon and rectum (JSCCR) guidelines 2019 for the treatment of colorectal cancer. Int J Clin Oncol 25: $1-42,2020$

3. WHO Classification of Tumours Editorial Board: Digestive System Tumours: WHO Classification of Tumours. IARC Publications, Lyon, 2019.

4. McGranahan N and Swanton C: Clonal heterogeneity and tumor evolution: Past, present, and the future. Cell 168: 613-628, 2017

5. Mukai M, Kishima K, Fukumitsu H, Sekido Y, Izumi H, Hoshikawa T, Tajima T, Tobita K, Sadahiro S, Yasuda S and Ogoshi K: Is the T1/2N1 ( $\leq 3$ nodes) category actually stage IIIA (TNM)/IIIa (Japanese classification) in patients with primary colorectal cancer? Oncol Rep 26: 209-214, 2011.

6. World Health Organization (WHO): International statistical classification of diseases and health related problems. ICD-10. WHO, Geneva, 2013.

7. Japanese Society for Cancer of the Colon and Rectum (JSCCR): Japanese Classification of Colorectal Carcinoma. Kanehara \& Co., Ltd., Tokyo, pp10-32, 2013.

8. National Comprehensive Cancer Network (NCCN): NCCN clinical practice guidelines in oncology rectal cancer. Version 3.2017. NCCN, Plymouth Meeting, PA, 2017.

9. National Comprehensive Cancer Network (NCCN): NCCN clinical practice guidelines in oncology colon cancer. Version 2.2017, 2017. NCCN, Plymouth Meeting, PA, 2017.

10. Tsakraklides V, Wanebo HJ, Sternberg SS, Stearns M and Good RA: Prognostic evaluation of regional lymph node morphology colorectal cancer. Am J Surg 129: 174-180, 1975.

11. Schofield JB, Mounter NA, Mallett R and Haboubi NY: The importance of accurate pathological assessment of lymph node involvement in colorectal cancer. Colorectal Dis 8: 460-470, 2006.

12. Shanmugam C, Hines RB, Jhala NC, Katkoori VR, Zhang B, Posey JA Jr, Bumpers HL, Grizzle WE, Eltoum IE, Siegal GP and Manne U: Evaluation of lymph node numbers for adequate staging of stage II and III colon cancer. J Hematol Oncol 4: 25, 2011

13. Jiang K, Zhu Y, Liu Y, Ye Y, Xie Q, Yang X and Wang S: Lymph node ratio as an independent prognostic indicator in stage III colorectal cancer: especially for fewer than 12 lymph nodes examined. Tumour Biol 35: 11685-11690, 2014.

14. Derwinger K, Carlsson G and Ekman T: Defining stage III disease in colorectal cancer-aspects on treatment and evaluation of survival. J Surg Oncol 102: 424-427, 2010.

15. Wang LP, Wang HY, Cao R, Zhu C and Wu XZ: Proposal of a new classification for stage III colorectal cancer based on the number and ratio of metastatic lymph nodes. World J Surg 37: 1094-1102, 2013

16. Wong KP, Poon JT, Fan JK and Law WL: Prognostic value of lymph node ratio in stage III colorectal cancer. Colorectal Dis 13: 1116-1122, 2011

17. Basnet S, Lou QF, Liu N, Rana R, Shah A, Khadka M, Warrier H, Sigdel S, Dhakal S, Devkota A, et al: Tumor deposit is an independent prognostic indicator in patients who underwent radical resection for colorectal cancer. J Cancer 9: 3979-3985, 2018.

18. Nagtegaal ID, Knijn N, Hugen N, Marshall HC, Sugihara K, Tot T, Ueno H and Quirke P: Tumor deposits in colorectal cancer: Improving the value of modern staging-a systematic review and meta-analysis. J Clin Oncol 35: 1119-1127, 2017.

19. Nagayoshi K, Ueki T, Nishioka Y, Manabe T, Mizuuchi Y, Hirahashi M, Oda Y and Tanaka M: Tumor deposit is a poor prognostic indicator for patients who have stage II and III colorectal cancer with fewer than 4 lymph node metastases but not for those with 4 or more. Dis Colon Rectum 57: 467-474, 2014.

20. Laso CA, González JJ, Fresno F, Azcano E, Sanz L and Navarrete F: Prognostic value of micrometastases in esophageal and colorectal carcinoma (a clinical experience). Hepatogastroenterology 51: 964-967, 2004.

21. Mescoli C, Albertoni L, Pucciarelli S, Giacomelli L, Russo VM, Fassan M, Nitti D and Rugge M: Isolated tumor cells in regional lymph nodes as relapse predictors in stage I and II colorectal cancer. J Clin Oncol 30: 965-971, 2012. 
22. Onaka T, Tsunoda A, Watanabe M, Nakao K, Takimoto M and Kusano M: The pathway of isolated tumor cells from tumor budding in stage II colorectal cancer detected by immunohistochemistry. Showa Univ J Med Sci 21: 25-36, 2009.

23. Lee MR, Hong CW, Yoon SN, Lim SB, Park KJ, Lee MJ, Kim WH and Park JG: Isolated tumor cells in lymph nodes are not a prognostic marker for patients with stage I and II colorectal cancer. J Surg Oncol 93: 13-19, 2006.

24. Mukai M, Sato S, Komatsu N, Nishida T, Shiba K, Ito I, Nakasaki H and Makuuchi H: Correlation between occult neoplastic cells in the lymph node sinuses and recurrence in patients with Dukes' C colorectal cancer. Oncol Rep 10: 1165-1169, 2003.

25. Mukai M, Sato S, Komatsu N, Nishida T, Shiba K, Ito I, Nakasaki $\mathrm{H}$ and Makuuchi $\mathrm{H}$ : Correlation between occult neoplastic cells in the lymph node sinuses and recurrence in patients with curatively resected Dukes' B colorectal cancer. Oncol Rep 10: 1177-1181, 2003.

26. Mukai M, Sato S, Nishida T, Komatsu N, Shiba K, Nakasaki H and Makuuchi H: Selection criteria for high risk and low risk groups of recurrence and metastasis in patients with primary colorectal cancer. Oncol Rep 10: 1753-1758, 2003.

27. Mukai M, Sato S, Nakasaki H, Tajima T, Saito Y, Nishiumi N, Iwasaki M, Tokuda Y, Ogoshi K, Inoue H and Makuuchi H: Occult neoplastic cells in the lymph node sinuses and recurrence of primary breast, lung, esophageal, and gastric cancer. Oncol Rep 11: 81-84, 2004.

28. Mukai M: Occult neoplastic cells and malignant micro-aggregates in lymph node sinuses: Review and hypothesis. Oncol Rep 14: 173-175, 2005.

29. Sekido Y, Mukai M, Kishima K, Tajima T, Hoshikawa T, Nakamura M, Nakamura N and Ogoshi K: Occult neoplastic cells in the lymph node sinuses and recurrence/metastasis of stage II/Dukes' B colorectal cancer. Oncol Rep 25: 69-73, 2011.
30. Sekido Y, Mukai M, Kishima K, Tajima T, Hoshikawa T, Nakamura M, Nakamura N and Ogoshi K: Occult neoplastic cells in the lymph node sinuses and recurrence/metastasis of stage III/Dukes' C colorectal cancer. Oncol Rep 25: 915-919, 2011.

31. Nakamura Y, Mukai M, Hiraiwa S, Kishima K, Sugiyama T, Tajiri T, Yamada S and Iwazaki M: Free-floating cancer cells in lymph node sinuses of hilar lymph node-positive patients with non-small cell lung cancer. Mol Med Rep 18: 1081-1087, 2018.

32. Romiti A, Roberto M, Marchetti P, Di Cerbo A, Falcone R, Campisi G, Ferri M, Balducci G, Ramacciato G, Ruco L and Pilozzi E: Study of histopathologic parameters to define the prognosis of stage II colon cancer. Int J Colorectal Dis 34: 905-913, 2019.

33. Compton CC: Colorectal carcinoma: Diagnostic, prognostic, and molecular features. Mod Pathol 16: 376-388, 2003.

34. Gunderson LL, Jessup JM, Sargent DJ, Greene FL and Stewart A: Revised tumor and node categorization for rectal cancer based on surveillance, epidemiology, and end results and rectal pooled analysis outcomes. J Clin Oncol 28: 256-263, 2010.

35. Shankaran V, Ikeda H, Bruce AT, White JM, Swanson PE, Old LJ and Schreiber RD: IFNgamma and lymphocytes prevent primary tumour development and shape tumour immunogenicity. Nature 410: 1107-11, 2001.

This work is licensed under a Creative Commons Attribution-NonCommercial-NoDerivatives 4.0 International (CC BY-NC-ND 4.0) License. 